\title{
POPULATION STUDY ARTICLE Maternal anemia type during pregnancy is associated with anemia risk among offspring during infancy
}

\author{
Ajibola I. Abioye ${ }^{1,2,3}$, Emily A. McDonald ${ }^{1,2,3}$, Sangshin Park ${ }^{1,2,3,4}$, Kelsey Ripp ${ }^{1,5,6}$, Brady Bennett ${ }^{2,7,8}$, Hannah W. Wu ${ }^{1,2,3}$, \\ Sunthorn Pond-Tor ${ }^{1,2,9}$, Marianne J. Sagliba ${ }^{10}$, Amabelle J. Amoylen ${ }^{10}$, Palmera I. Baltazar ${ }^{11}$, Veronica Tallo ${ }^{10}$, Luz P. Acosta ${ }^{10}$, \\ Remigio M. Olveda ${ }^{10}$, Jonathan D. Kurtis ${ }^{1,2,9}$ and Jennifer F. Friedman ${ }^{1,2,3}$
}

\begin{abstract}
BACKGROUND: We evaluated the association between etiology of maternal anemia and iron status throughout infancy. METHODS: Samples from a study designed to examine Praziquantel treatment during pregnancy were used $(n=359)$. All women were infected with schistosomiasis and randomized to Praziquantel or placebo at $16 \pm 2$ weeks' gestation. Hemoglobin, serum ferritin (SF), soluble transferrin receptor (sTfR), hepcidin, C-reactive protein, and interleukin-6 were measured in maternal and infant blood. The relationship between both maternal Praziquantel treatment and etiology of anemia and infant iron status was evaluated. RESULTS: Maternal iron-deficiency anemia was associated with increased risk of infant anemia at 6 months of age. Infants of mothers with the lowest levels of circulating hepcidin during gestation, likely a marker for iron deficiency, had higher sTfR:SF levels and lower hemoglobin levels, particularly at 12 months of age. Maternal non-iron-deficiency anemia (NIDA) did not impact infant anemia risk or iron status. Maternal treatment for schistosomiasis had no effect on infant hematologic status.

CONCLUSIONS: Maternal iron deficiency anemia was associated with an increased risk for anemia or iron deficiency during late infancy. We did not observe an association between maternal NIDA and increased risk for iron deficiency during infancy.
\end{abstract}

Pediatric Research (2019) 86:396-402; https://doi.org/10.1038/s41390-019-0433-5

\section{INTRODUCTION}

Anemia affects an estimated 273 million children under the age of 5 years throughout the world, with a disproportionate burden in low and middle income countries (LMICs). ${ }^{1}$ Maternal anemia during pregnancy has been associated with increased risk of maternal and fetal mortality, poor intrauterine growth, and impaired cognitive development in affected offspring. ${ }^{2}$ Importantly, in both human and animal models, short- and long-term neurodevelopmental deficits have been demonstrated to persist even after iron repletion..$^{3-6}$ Inadequate body iron is thought to account for approximately $50 \%$ of anemia in women globally (iron-deficiency anemia (IDA)). Additionally, non-iron-deficiency anemia (NIDA), related to other micronutrient deficiencies and/or infections with resulting anemia of inflammation (Al), is also a major contributor to anemia in this population. ${ }^{1,7}$

Owing to the importance of iron in neurocognitive development, ${ }^{3-6,8}$ identifying risk factors for infant iron deficiency is critical. Though the fetus is thought to efficiency obtain iron in utero, many studies have demonstrated lower cord hemoglobin and iron levels among infants of anemic women, particularly women with severe anemia. ${ }^{9,10}$ Maternal iron prophylaxis during gestation and newborn serum ferritin (SF) are both positive predictors of SF in infancy. ${ }^{11}$ Further, in a recently published study from The Philippines, we found that maternal IDA, but not NIDA (due largely to $\mathrm{Al}$ ), influenced newborn iron endowment. ${ }^{12}$ Less is known about the longitudinal effect of maternal IDA and Al, both of which may continue to influence infant iron status. Specifically, the impact of both maternal IDA and AI on infant iron status may not become evident until after in utero stores are depleted, usually around 4 months of age among term breastfed infants, ${ }^{13}$ and decreased iron bioavailability may continue to limit iron bioavailability in breast milk. ${ }^{10}$ No studies have examined the role of $\mathrm{Al}$ on longitudinal infant iron status. Given Al causes decreased iron bioavailability, we hypothesized that this process might impact infant iron status through decreased fetal and infant iron acquisition in utero and in breast milk.

Determining the etiology of maternal anemia and its impact on longitudinal infant iron status and anemia risk is important as interventions to reduce this risk differ significantly. In the context of $\mathrm{Al}$, hepcidin production, driven primarily by the proinflammatory cytokine, interleukin (IL)- $6^{14}$ leads to decreased iron absorption in the gut and iron sequestration in the reticuloendothelial system by causing the degradation of membrane-bound ferroportin. ${ }^{15}$ In the context of iron deficiency, hepcidin is typically suppressed to maintain ferroportin integrity, allowing increased iron absorption in the gut and release from macrophages. ${ }^{16}$ Maternal hepcidin has also been shown to be inversely related to iron bioavailability to the fetus. ${ }^{17}$

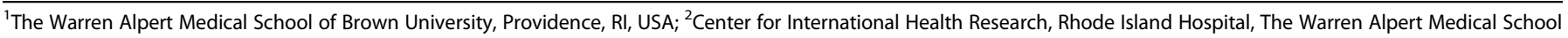

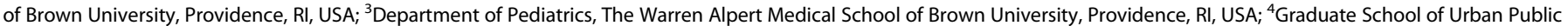

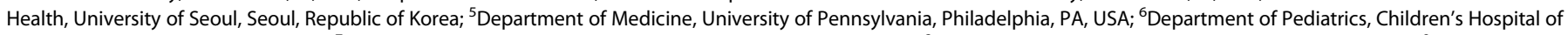

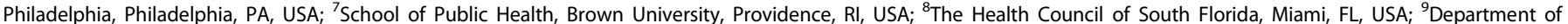

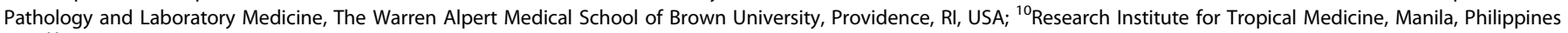
and ${ }^{11}$ Remedios Trinidad Romualdez Hospital, Tacloban City, Leyte, The Philippines

Correspondence: Sangshin Park (spark@uos.ac.kr)

These authors contributed equally: Ajibola I. Abioye, Emily A. McDonald.
}

Received: 12 June 2018 Revised: 5 February 2019 Accepted: 6 May 2019

Published online: 26 May 2019 
In previous work conducted among children and non-pregnant adults in Leyte, The Philippines, we demonstrated that Schistosoma japonicum infection is related to anemia risk across all infection intensities. ${ }^{18,19}$ Importantly, the predominant cause of anemia in this context is NIDA ( $20 \%$ overall prevalence), primarily due to $\mathrm{Al}$, with a prevalence of IDA of $12 \% .{ }^{18,19}$ Though the etiology of NIDA is multi-factorial, in those studies we found that $<1 \%$ of individuals with NIDA had a macrocytic anemia, making B12 or folate deficiency the primary cause of NIDA unlikely. S. japonicum infection in this population is believed to lead to Al via chronic immune activation, both in response to adult worms and trapped eggs, ${ }^{20}$ and as a result of microbial translocation across the gut epithelium as eggs penetrate the gut wall from the largely sterile bloodstream. ${ }^{21}$

The objectives of this study were to evaluate the impact of (1) the specific etiology of maternal anemia and (2) maternal treatment for $S$. japonicum on infant iron and anemia status and (3) examine the relationship between measures of maternal and newborn iron status and longitudinal anemia risk in infancy.

\section{SUBJECTS AND METHODS}

Study site and population

Our study population has been described elsewhere. ${ }^{22}$ The study was conducted in Leyte, The Philippines where S. japonicum is endemic. Geo-helminth infections were also prevalent. Briefly, all women were positive for schistosomiasis during the first trimester of pregnancy and randomized to Praziquantel treatment or placebo at 12-16 weeks gestation. All of the women were provided with a daily multivitamin with iron ( $29 \mathrm{mg}$ elemental $\mathrm{Fe}$ ) dispensed at enrollment and the 22- and 32-week visits. At the 22and 32 -week visits, we captured reported compliance, and $100 \%$ of study participants reported taking their prenatal vitamins as reported for the trial. ${ }^{22}$

Eligibility criteria included infection with $S$. japonicum, the presence of a singleton pregnancy in the second or third trimester, age $\geq 18$ years, and provision of informed consent. Of note, treatment did not impact birth weight, risk of low birth weight (LBW), or small for gestational age but did impact maternal ferritin at 32 weeks' gestation as reported. ${ }^{22}$

\section{Stool examination}

Maternal parasite burden was determined by examination of three consecutive stool specimens. Each of the three stool specimens was examined in duplicate for S. japonicum, Ascaris lumbricoides, Trichuris trichuria, and hookworm by the Kato Katz method. Intensity of infection for each helminth was determined using the World Health Organization (WHO) criteria. $^{23}$

\section{Blood collection and processing}

Venipuncture was performed at 32 weeks' gestation and for the infant at 6 and 12 months of age. A cord blood sample was also obtained at delivery. A complete hemogram was obtained on a Horiba $A B X$ hematology analyzer (Kyoto, Japan) for the infant samples, including mean corpuscular volume (MCV). SF and serum transferrin receptor (sTfR) were assessed using commercially available enzyme-linked immunosorbent assays (ELISAs) (R\&D Systems, Minneapolis, MN). C-reactive protein (CRP) and IL-6 were analyzed using a multiplex bead-based platform (BioRad, Hercules, CA) and custom sandwich- or competitivestyle ELISA assays. ${ }^{24}$ The reference ranges for sTfR and STfR-SF Index (=sTfR/ $\left.\log _{10}(\mathrm{SF})\right)$ based on the Quantikine assay kits from R\&D Systems were $12.16-27.25 \mathrm{nmol} / \mathrm{L}$ and 6.42-22.37 (using $\mathrm{nmol} / \mathrm{L}$ for sTfR and $\mathrm{ng} / \mathrm{mL}$ for SF), respectively. ${ }^{25}$ Hepcidin was measured using a commercially available ELISA Kit (DRG Diagnostics, Marburg, Germany). The reference range $(2.5-97.5 \%$ ile) among healthy non-pregnant subjects is 1.49-41.46. Participants with extremely low undetectable concentrations of biomarkers were assigned the lowest detectable concentrations.

Assessment of newborn outcomes

Gestational age at birth was based on obstetric ultrasound at 8-12 weeks gestation. The newborn was weighed on a Tanita model BD-585 (Arlington Heights, MD) portable scale within $24 \mathrm{~h}$ of birth. This digital scale measures birth weight with precision to $10 \mathrm{~g}$.

Anemia and iron status

Infant anemia was defined as hemoglobin concentration $<11.0 \mathrm{~g} /$ $\mathrm{dL}^{26}$ Infant IDA was identified using ferritin concentration $<12 \mu \mathrm{g} /$ $L$ in the absence of inflammation (CRP $\leq 5 \mathrm{mg} / \mathrm{L}$ ) or ferritin $<30 \mu \mathrm{g} /$ $L$ in the presence of inflammation (CRP $>5 \mathrm{mg} / \mathrm{L}$ ). ${ }^{27}$ Infants who were anemic but not iron-deficient were regarded as having NIDA. Maternal anemia in the presence of ferritin $<30 \mu \mathrm{g} / \mathrm{L}$ was used to define maternal IDA, and anemia with ferritin level $\geq 30 \mu \mathrm{g} / \mathrm{L}$ to define maternal NIDA. ${ }^{28}$ We used the sTfR-SF Index as the primary measure of infant iron status, given this ratio is less affected by inflammation ${ }^{29}$ and has been shown to reflect bone marrow iron stores in children. ${ }^{30}$ Red cell macrocytosis was defined as an MCV $>100 \mathrm{fL}$.

\section{Statistical analysis}

We examined the relationship between two primary predictors (maternal treatment status as Praziquantel versus placebo and the type of maternal anemia as no anemia, IDA, or NIDA) and three primary outcomes (infant iron status, hemoglobin, and anemia status). Analyses were conducted using SAS 9.3 (SAS Institute, Cary, NC). Statistical significance was defined as $P<0.05$. Concentrations of ferritin, IL-6, CRP, sTfR, and hepcidin and the sTfR-SF Index were skewed and were, therefore, natural logtransformed for regression analyses. The following confounding and mediating covariates were evaluated for inclusion in regression models if they were related to the outcomes of interest in bivariate analyses with $P<0.2$ : newborn sex; LBW; preterm birth; birth length; maternal educational attainment; parity; socioeconomic status; maternal obesity; maternal alcohol consumption; maternal smoking; current breastfeeding at months 1, 6, and 12; infant nutritional status at 6 and 12 months; and maternal infection with $A$. lumbricoides, hookworm, or $T$. trichiura at 32 weeks' gestation. Maternal age and treatment were included in all models. Log-binomial regression models were used to evaluate the association of maternal anemia type at 32 weeks' gestation with the risk of infant anemia at 6 and 12 months. Generalized estimating equations models were used to evaluate the association of the change in hemoglobin from 6 to 12 months across maternal hepcidin at 32 weeks' gestation and cord blood hepcidin concentration, in tertiles. Linear regression models were used to evaluate the association of maternal and cord blood hepcidin tertiles with infants' hematologic biomarker concentrations at each time point.

\section{Ethical clearance and informed consent}

The institutional review boards of Rhode Island Hospital and The Research Institute of Tropical Medicine approved this study. Written informed consent was obtained from each participant. All women were offered treatment for $S$. japonicum and geohelminths following pregnancy and lactation, in accordance with Department of Health policy in the Philippines at the time of the study.

\section{RESULTS}

Characteristics of the 359 mother-infant pairs (Fig. 1) stratified by maternal treatment status are outlined in Table 1. The distribution of maternal biomarkers at 32 weeks' gestation is also presented in 


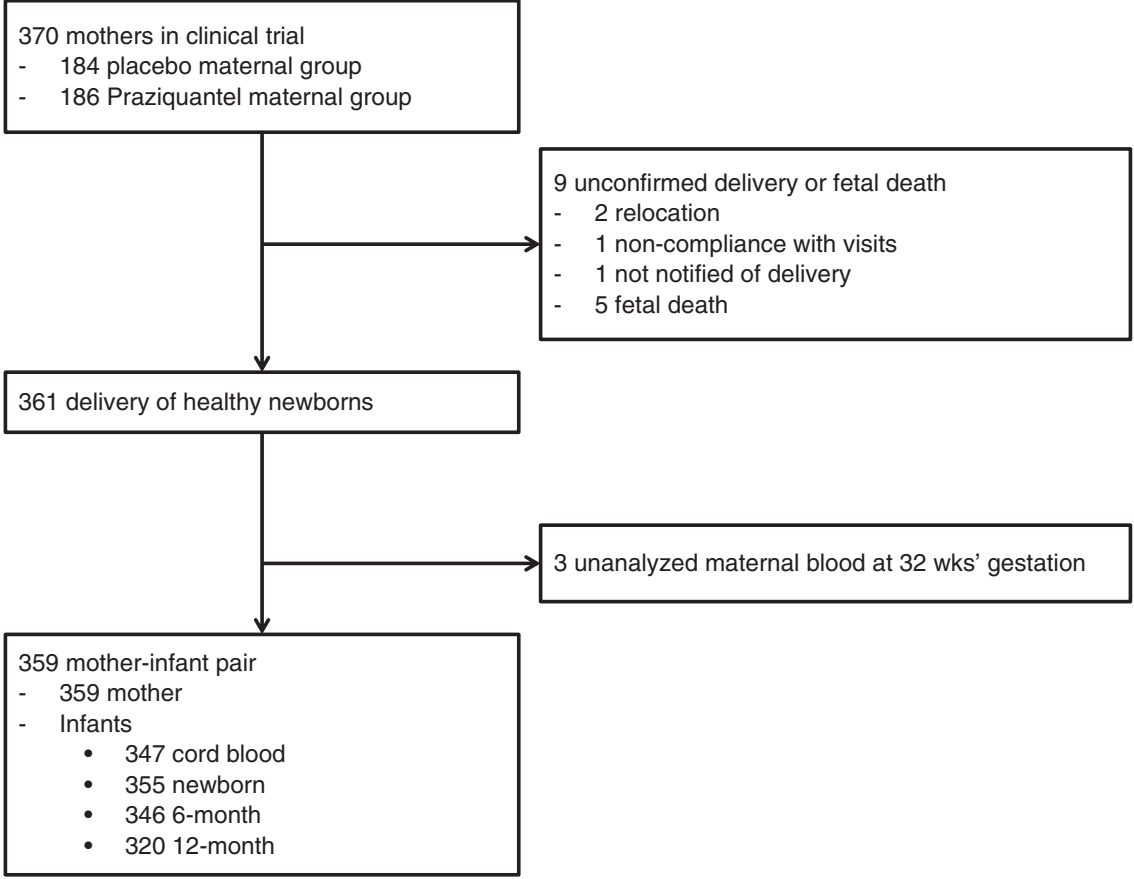

Fig. 1 Study participants

Table 2. The majority of infants were born at term, with a mean birth weight of $2.85 \mathrm{~kg}$. Geo-helminth infections were also prevalent. Hemoglobin and iron status at 6 and 12 months of age were similar among infants born to mothers who were treated with Praziquantel, compared to those the placebo group. At both 6 and 12 months of age, the prevalence of infant anemia was high ( $66 \%$ and $64 \%$, respectively). At 6 months of age, $45 \%$ of infants had IDA and this increased to $51 \%$ at 12 months of age. At 6 and 12 months of age, $21 \%$ and $13 \%$ of infants had NIDA, respectively (Table 1). Of note, only 1 infant at 6 months of age had an MCV $<$ $100 \mathrm{fL}$.

Infant anemia status is associated with maternal anemia status during gestation

Infant anemia prevalence differed significantly by the type of maternal anemia during pregnancy (Fig. 2). At 6 months of age, infants of mothers with IDA at 32 weeks' gestation had significantly higher anemia risk, compared to infants of mothers with NIDA $(P<0.006)$. Interestingly, infants of women with NIDA had the lowest anemia prevalence at 6 months of age. Infant anemia prevalence at 12 months of age was no longer related to maternal anemia status $(P=0.78)$. The prevalence of infant anemia did not differ significantly between 6 and 12 months of age and was relatively high across the entire cohort at both time points (Table 1, Fig. 2).

Maternal hepcidin is associated with infant iron status Infants of mothers in the lowest tertile of 32-week hepcidin levels had a significantly higher median STfR-SF Index at 12 months of age, reflecting decreasing iron status, compared to infants of the same age born to mothers with the highest levels of hepcidin (Fig. 3a). Among mothers with the lowest levels of circulating hepcidin at 32 weeks' gestation, infants displayed a significant increase in the median sTfR-SF Index, from 6 to 12 months of age (Fig. 3a). Interestingly, we observed no differences in iron status in 6-month-old infants based on maternal hepcidin levels. Cord blood hepcidin concentration was not associated with the sTfR-SF Index at 6 or 12 months and the change in STfR-SF Index from 6 to 12 months did not differ by cord blood hepcidin tertile (Fig. 3b).

Maternal hepcidin is associated with infant hemoglobin levels There were no significant differences in infant hemoglobin concentrations at 6 months across maternal hepcidin tertiles. Infants from mothers in the lowest hepcidin tertile during gestation, however, had significantly lower hemoglobin concentration at 12 months of age $(P<0.01)$ compared to those with moderate hepcidin levels (Fig. 4a). These infants also had a significant decrease in hemoglobin concentration from 6 to 12 months $(P<0.05)$, while there were no significant changes in hemoglobin concentration among infants in the other maternal hepcidin tertiles (Fig. 4a). There was no association between cord blood hepcidin tertiles and infant hemoglobin concentration at 6 or 12 months of age (Fig. 4b).

Infant anemia type and iron and inflammatory biomarkers The pro-inflammatory cytokine IL-6 was higher in infants with NIDA compared to infants with IDA $(P=0.027)$ at both ages and higher in infants with NIDA compared to infants without anemia $(P=0.036)$ at 12 months (Fig. 5a, e). As expected, hepcidin concentrations were lower in infants with IDA at both 6 and 12 months compared to infants with NIDA ( $P<0.01$; Fig. 5b, f), and lower in IDA compared to non-anemic children only at 12 months of age ( $P<0.05$, Fig. $5 f)$. In addition, despite significantly higher IL6 levels in NIDA infants compared to their non-anemic counterparts, hepcidin levels were not significantly different between these two groups at 12 months of age (Fig. 5f). Conversely, sTfR concentrations were the highest in IDA infants compared to either non-anemic or NIDA counterparts of the same age $(P<0.01$, Fig. $5 c, g)$. CRP levels were not significantly different based on infant anemia status at either time point (Fig. $5 \mathrm{~d}$, h). Overall, while STfR and STfR-SF Index increased over time (6 months compared to 12 months: $P<0.01)$ and across all groups, ferritin $(P=0.002)$ and hepcidin decreased $(P=0.001)$, reflecting a downward trend in iron status in late infancy (data not shown). The concentrations of hemoglobin did not change significantly $(P=0.68)$ over time, 
Table 1. Characteristics of the study population, stratified by maternal treatment status

\begin{tabular}{|c|c|c|c|}
\hline & Praziquantel $(n=179)$ & Placebo $(n=180)$ & Total $(n=359)$ \\
\hline Non-anemic, $n(\%)$ & $99(55)$ & $92(51)$ & $191(53)$ \\
\hline NIDA, $n(\%)$ & $18(10)$ & $14(8)$ & $32(9)$ \\
\hline Hemoglobin, g/dL, median (IQR) & $11(10.2,11.8)$ & $11(10,11.9)$ & $11(10.1,11.8)$ \\
\hline Ferritin, $\mu \mathrm{g} / \mathrm{L}$, median (IQR) & $14.7(2.8,28.4)$ & $10.5(2.8,21.8)$ & $12.1(2.8,25.3)$ \\
\hline$M C V,>100 \mathrm{fL}, \%$ & $1(1)$ & $4(2)$ & $5(1)$ \\
\hline \multicolumn{4}{|l|}{ Infant, birth, $n=359$} \\
\hline Male, $n(\%)$ & $97(54)$ & $94(52)$ & $191(53)$ \\
\hline Birth weight $(\mathrm{g})$; mean $(95 \% \mathrm{Cl})$ & $2.85(2.78-2.91)$ & $2.85(2.79-2.91)$ & $2.85(2.81-2.89)$ \\
\hline Gestational age (weeks); median (IQR) & $39(38-39)$ & $38.7(37.9-39.4)$ & $38.7(38.0,39.4)$ \\
\hline IDA, $n(\%)$ & $91(57)$ & $75(47)$ & $166(52)$ \\
\hline NIDA, $n(\%)$ & $20(13)$ & $24(15)$ & $44(14)$ \\
\hline Hemoglobin, g/dL, median (IQR) & $10.4(9.5,11.2)$ & $10.4(9.6,11.6)$ & $10.4(9.5,11.3)$ \\
\hline Ferritin, $\mu \mathrm{g} / \mathrm{L}$, median (IQR) & $9.5(1.6,24.5)$ & $10.0(2.5,28.5)$ & $9.5(1.8,26.8)$ \\
\hline Hepcidin, $\mu \mathrm{g} / \mathrm{L}$, median (IQR) & $11.0(5.6,21.8)$ & $10.6(6.1,19)$ & $10.8(6.0,21.3)$ \\
\hline sTfR, mg/L, median (IQR) & $1.9(1.6,2.3)$ & $1.8(1.5,2.3)$ & $1.9(1.6,2.3)$ \\
\hline CRP, mg/L median (IQR) & $3.1(0.9,8.6)$ & $2.1(0.8,6.9)$ & $2.6(0.8,7.3)$ \\
\hline sTfR:log(ferritin), median (IQR) & $1.6(1.2,2.2)$ & $1.5(1.0,2.2)$ & $1.5(1.1,2.2)$ \\
\hline MCV, fL, median & $71(67,75)$ & $71(66,75)$ & $71(66,75)$ \\
\hline$M C V,>100 f L, \%$ & $1(1)$ & $0(0)$ & $1(0.3)$ \\
\hline \multicolumn{4}{|l|}{ Infant, 12 months of age, $n=312$} \\
\hline sTfR, mg/L median (IQR) & $2.1(1.7,2.7)$ & $2.1(1.8,2.8)$ & $2.1(1.7,2.7)$ \\
\hline CRP, mg/L, median (IQR) & $3.1(0.8,8.0)$ & $1.5(0.5,6.1)$ & $2.5(0.7,6.5)$ \\
\hline sTfR:log(ferritin), median (IQR) & $2.0(1.4,2.9)$ & $1.9(1.4,3.0)$ & $1.9(1.4,2.9)$ \\
\hline $\mathrm{MCV}, \mathrm{fL}$, median & $69(64,75)$ & $70(64,74)$ & $70(64,74)$ \\
\hline$M C V,>100 \mathrm{fL}, \%$ & $0(0)$ & $0(0)$ & $0(0)$ \\
\hline
\end{tabular}

The influence of Praziquantel on population characteristics was assessed using chi-square tests for categorical variables and Wilcoxon tests for continuous variables. None of the characteristics evaluated were significantly different by treatment status

$C l$ confidence interval, CRP C-reactive protein, IDA iron-deficiency anemia, IQR interquartile range, MCV mean corpuscular volume, NIDA non-iron-deficiency anemia, sTfR soluble transferrin receptor

Table 2. Characteristics of mothers by anemia type at 32 weeks' of gestation

\begin{tabular}{lccc}
\hline Maternal, 32 weeks' gestation & IDA $(n=136)$ & NIDA $(n=32)$ & No anemia $(n=191)$ \\
\hline Hemoglobin, $\mathrm{g} / \mathrm{dL}$ & $10.0 \pm 0.1$ & $10.1 \pm 0.1$ & $11.9 \pm 0.1$ \\
Serum ferritin, $\mu \mathrm{g} / \mathrm{L}$ & $5.7(0.7-16.4)$ & $46.7(37.7-72.3)$ & $13.9(11.6-15.5)$ \\
Serum hepcidin, $\mu \mathrm{g} / \mathrm{L}$ & $2.5(0.9-4.9)$ & $6.2(3.4-10.5)$ & $2.8(1.1-5.6)$ \\
Serum CRP, $\mathrm{mg} / \mathrm{L}$ & $3.9(2.0-8.1)$ & $7.3(2.2-21.7)$ & $4.3(1.9-8.4)$ \\
MCV, $\mathrm{fL}$ & $86.5(81.0-90.0)$ & $89.5(86.0-92.0)$ & $89.0(86.0-93.0)$ \\
\hline
\end{tabular}

CRP C-reactive protein, IDA iron-deficiency anemia, MCV mean corpuscular volume, NIDA non-iron-deficiency anemia 
likely reflecting the order in which these indices of iron status change.

\section{DISCUSSION}

In this study, we evaluated the influence of maternal anemia type and gestational treatment for schistosomiasis on infant iron status and anemia risk. In our previous studies in non-pregnant subjects, we have demonstrated that schistosomiasis is associated with Al. ${ }^{18,19}$ Though treated mothers demonstrated improved iron stores, and their newborns displayed modest improvements in iron endowment, ${ }^{22}$ in the present analysis we were unable to identify an association between maternal treatment and iron status or anemia risk during later infancy. This may in part be due to prolonged pro-inflammatory response to dying worms and eggs following Praziquantel administration ${ }^{31}$ and the improvement in hemoglobin in non-pregnant subjects after treatment does not occur for 3-6 months. ${ }^{32}$ We did, however, identify a significant relationship between maternal IDA, but not NIDA, during pregnancy and infant anemia risk at 6 months of age, as well as maternal iron status biomarkers and infant anemia risk.

Understanding the influence of maternal anemia type on infant iron deficiency and anemia risk is critical to successfully preventing and treating anemia among young children. ${ }^{33}$ Improved survival in early infancy has been shown among neonates and infants of mothers who received iron supplementation in multiple LMICs, often in a dose-responsive fashion. ${ }^{34,35}$ Given that IDA and Al require different approaches to treatment,

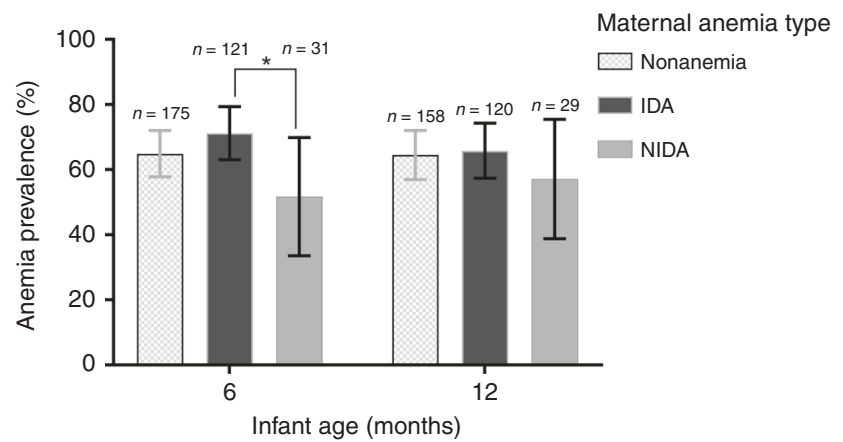

Fig. 2 Prevalence of infants' anemia at 6 months is highest in infants born to mothers who had iron deficiency anemia during gestation. Error bars represent $95 \%$ confidence intervals. ${ }^{*} P<0.05$ clarifying the extent to which maternal anemia type and iron status affects infant anemia risk is therefore critical.

We found that infants were more likely to be anemic if their mothers had IDA versus NIDA during pregnancy, despite the fact that all women were provided with pre-natal vitamins with iron. This association, however, is lost by 12 months of age, and overall rates of anemia in this infant population increase. It is possible that many of the infants at 12 months of age have pre-anemic iron deficiency, given demonstrated decreases in ferritin from 6 to 12 months of age without significant increase in anemia risk. Although the maternal-fetal dyad is adapted to maximize fetal iron uptake during gestation, ${ }^{36}$ infant iron stores may wane, particularly when breast milk is iron poor, by 6 months of age. ${ }^{37}$ The relatively high rates of iron deficiency in this pediatric population at both 6 and 12 months may also be reflective of the relative poor nutritional quality of complementary foods introduced during infancy in this population. Alterations in iron metabolism during pregnancy as seen in Al did not impact newborn iron status or anemia risk, suggesting that this mechanism does not significantly impact iron transfer. ${ }^{12}$

Interestingly, we found that maternal hepcidin, but not newborn hepcidin, was related to infant iron status and hemoglobin. In addition, low maternal hepcidin was associated with a significant worsening of infant iron status from 6 to 12 months. Hepcidin is a key regulator of iron bio-availability and is upregulated in response to inflammatory cytokines, particularly $\mathrm{IL}-6$, and downregulated in the context of iron deficiency. ${ }^{14-16} \mathrm{It}$ is likely that the relationship between low maternal hepcidin and increased risk for both infant iron deficiency and anemia is due to the fact that hepcidin is a marker for women with more significant iron deficiency, who may be less able to transfer sufficient iron to the fetus in utero and in breast milk to maintain stores at 12 months of age. This is further supported by the worsening iron status and hemoglobin from 6 to 12 months of age only among infants whose mothers had the lowest hepcidin levels. This may also be partly explained by a shared maternal-infant environment with iron-poor solid foods as the infant transitions to the addition of complementary foods.

In addition, we found a high prevalence of NIDA at both 6 and 12 months of age. Hematologic and inflammatory biomarker dynamics may reflect the etiology of anemia among infants. The MCV, elevated in the context of megaloblastic anemias due to B12 and folate deficiency, was only elevated in $0.3 \%$ of infants at 6 and 12 months of age, suggesting that these micronutrient deficiencies were not a significant cause of NIDA. IL- 6 concentrations at 6 and 12 months were higher among infants with NIDA, compared
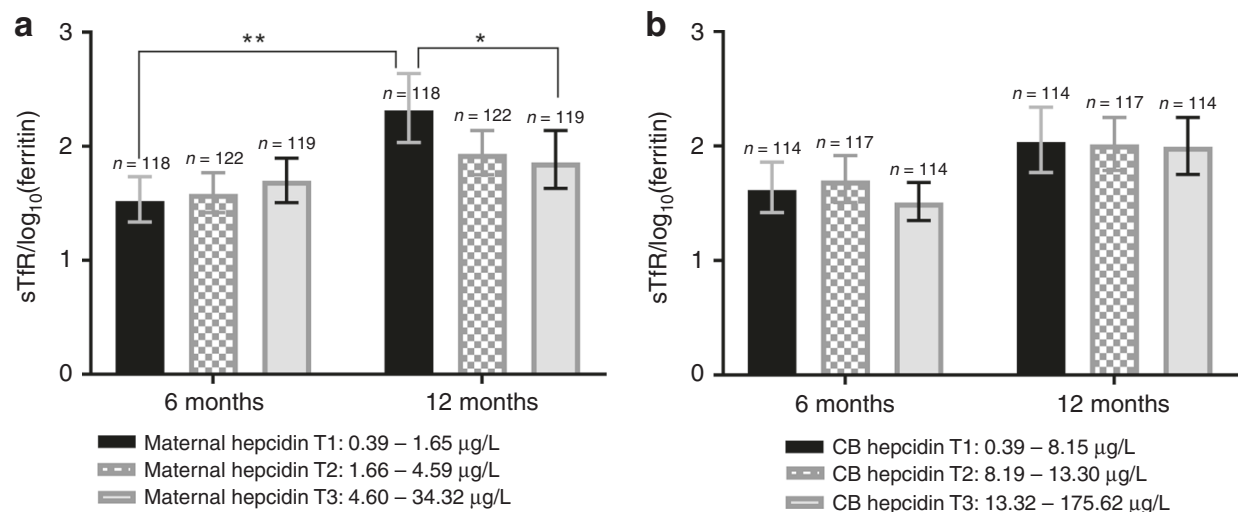

Fig. 3 Maternal hepcidin (a) but not newborn hepcidin (b) is related to infant iron status at 12 months of age. a Infants of mothers with the lowest hepcidin during pregnancy displayed the highest soluble transferrin receptor (sTfR)-ferritin index, and thus the lowest iron status, at 12 months of age. Infants born to those mothers with hepcidin levels in the lowest tertile had significantly higher sTfR-ferritin index values at 12 months compared to 6 months of age. b Fetal hepcidin levels were not related to infant iron status at either 6 or 12 months of age. CB cord blood; error bars represent the $95 \%$ confidence intervals. ${ }^{*} P<0.05 ;{ }^{* *} P<0.01$ 

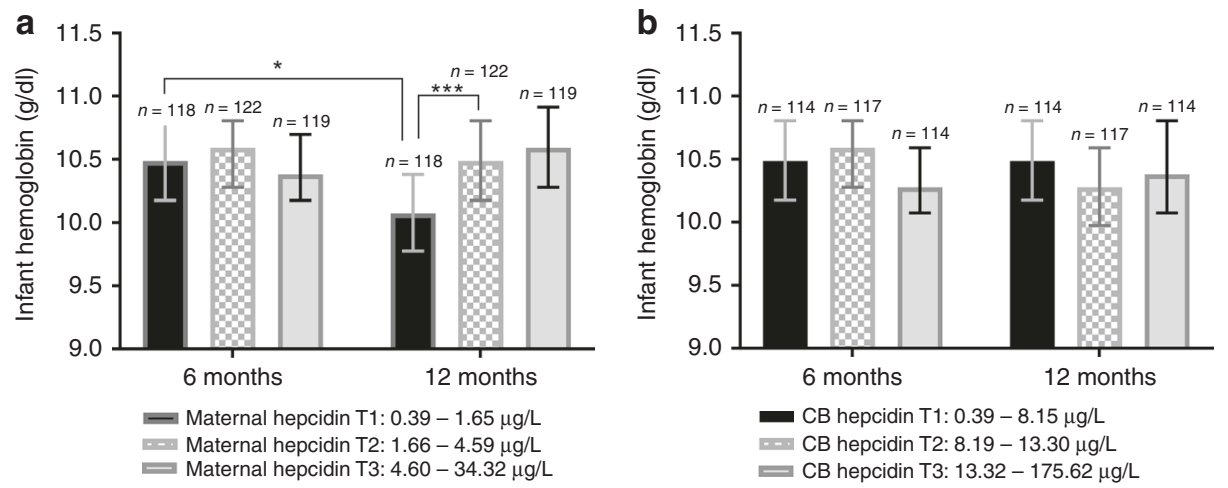

Fig. 4 Maternal circulating hepcidin (a) but not fetal hepcidin (b) is related to infant hemoglobin concentration at 12 months of age. a Infants from mothers with the lowest hepcidin during pregnancy displayed the lowest hemoglobin levels at 12 months of age. Infants born to those mothers with hepcidin levels in the lowest tertile had significantly lower hemoglobin values at 12 months compared to 6 months of age. b Newborn hepcidin levels were not related to infant hemoglobin at either 6 or 12 months of age. CB cord blood; error bars represent the $95 \%$ confidence intervals. ${ }^{*} P<0.05 ;{ }^{* * *} P<0.001$
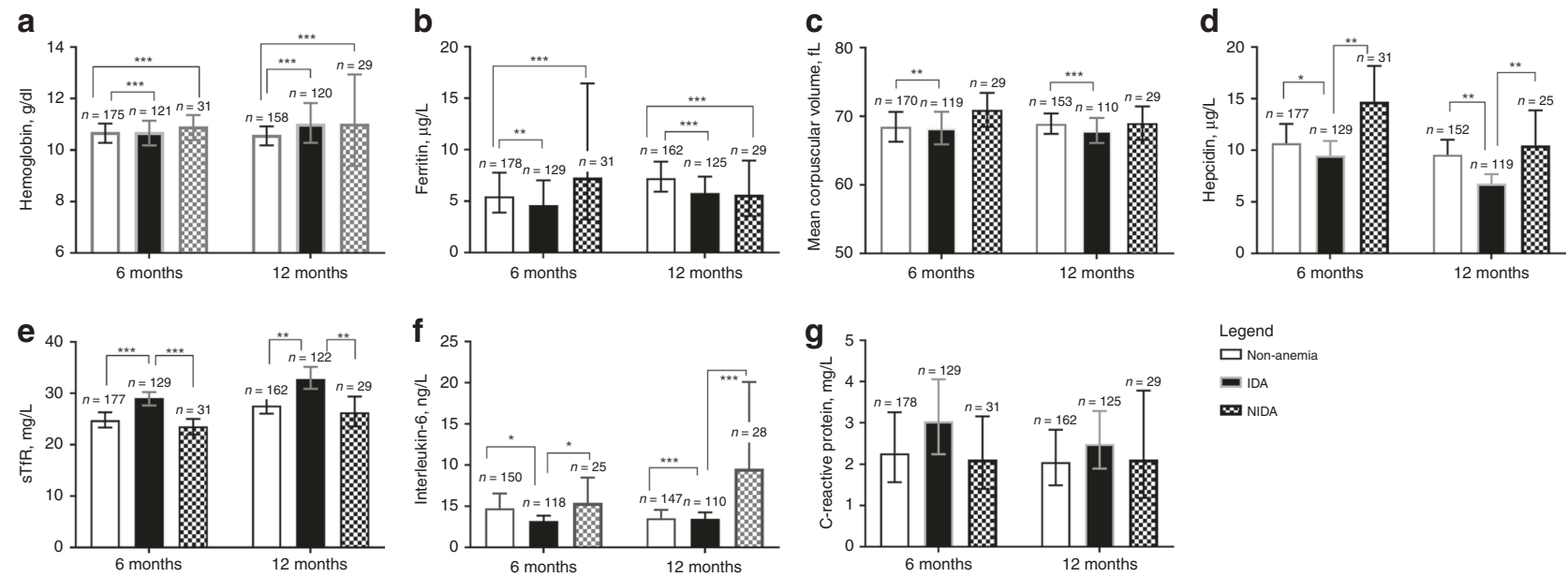

Fig. 5 Hematologic and inflammatory biomarker concentrations are related to infants' anemia type at 6 and 12 months of age. Interleukin (IL)6 and hepcidin are lower and soluble transferrin receptor (sTfR) is higher in 6-month-old infants with -iron deficiency anemia compared to others. This trend continues at 12 months of age. Further, IL-6 and hepcidin are higher and sTfR lower in 6-month-old infants with non-iron deficiency anemia compared to non-anemic infants at 6 and 12 months, respectively. Error bars represent $95 \%$ confidence intervals. Asterisk symbols ${ }^{*},{ }^{* *}$ and ${ }^{* * *}$ represent $P<0.05, P<0.01$, and $P<0.001$, respectively, and compare the concentration of each biomarker by anemia type

to IDA, and hepcidin was significantly higher among infants with NIDA compared to IDA at 6 and 12 months. Together, these findings suggest that NIDA in most of these infants is driven by Al.

Limitations to this study include a lack of standardized definitions to define the etiology of anemia during pregnancy and infancy. We used hemoglobin and ferritin to define maternal anemia types based on bone marrow studies ${ }^{28}$ and the WHO definition for infant anemia type. ${ }^{27}$ Unfortunately, we did not capture timing of the introduction of solid foods, thus limiting our ability to make conclusive statements regarding the impact of complementary foods on iron status. In addition, we did not assess infants for hemoglobinopathies, which may also cause NIDA, so we cannot determine the contribution of hemoglobinopathies or other less common causes of NIDA. Finally, we do not have a measure of maternal iron status or hemoglobin at the time of delivery. Given the importance of third trimester iron transfer, a more proximate measure of maternal iron status peri-natally might have changed maternal classification with respect to iron status and anemia type. Serum iron was also not measured in this study, although quantitations of STfR and ferritin have been shown to be excellent markers for iron status.
Iron status during infancy is likely influenced by both newborn iron endowment and the availability of iron during infancy in breast milk and complementary foods. ${ }^{38}$ Indeed, other groups have shown that exclusive breastfeeding improves iron status of infants in resource-poor settings. ${ }^{39}$ Our finding that maternal IDA continues to influence infant iron deficiency and anemia during infancy supports the importance of maternal iron nutriture and the need for improved pre-conceptual maternal iron status and better compliance with prenatal vitamins with iron, as well as continued provision of multivitamins with iron post-natally. The high prevalence of Al among infants highlights the high infection and inflammatory burden experienced by these infants and its contribution to anemia.

\section{ACKNOWLEDGEMENTS}

We thank our study participants, dedicated field staffs in Leyte, The Philippines and our funders (NIH/NIAID and Thrasher Research Fund) for supporting this work. The randomized controlled trial was supported by NIH/NIAID, "S. japonicum and pregnancy outcomes: An RCT" (U01Al066050) with relevant data for this manuscript collected through newborn day of life 28. NIH/NIAID (R21Al107520) "S. japonicum, anemia, and iron transport in human pregnancy" supported extended biomarkers to define etiology of anemia among pregnant women and their offspring. 


\section{AUTHOR CONTRIBUTIONS}

All the authors participated in the planning and design of the study. M.J.S., A.J.A., P.I. B., V.T., L.P.A. and R.M.O. participated in data collection. E.A.M., H.W.W., S.P.-T., and J.D. K. did the laboratory assays. A.I.A. and S.P. analyzed data. A.I.A., E.A.M. and J.F.F. participated in the primary manuscript writing. J.F.F. had primary responsibility for the final content. All authors read and approved the final manuscript.

\section{ADDITIONAL INFORMATION}

Competing interests: The authors declare no competing interests.

Publisher's note: Springer Nature remains neutral with regard to jurisdictional claims in published maps and institutional affiliations.

\section{REFERENCES}

1. Stevens, G. et al. Global, regional, and national trends in haemoglobin concentration and prevalence of total and severe anaemia in children and pregnancy and non-pregnant women for 1995-2011: a systematic analysis of populationrepresentative data. Lancet Glob. Health 1, e16-e25 (2013).

2. Menon, K. et al. Effects of anemia at different stages of gestation on infant outcomes. Nutrition 32, 61-65 (2016).

3. Clardy, S. L. et al. Acute and chronic effects of developmental iron deficiency on mRNA expression patterns in the brain. J. Neural Transm. Suppl. 173-196 (2006).

4. de Deungria, M. et al. Perinatal iron deficiency decreases cytochrome c oxidase (CytOx) activity in selected regions of neonatal rat brain. Pediatr. Res. 48, 169-176 (2000).

5. Felt, B. T. \& Lozoff, B. Brain iron and behavior of rats are not normalized by treatment of iron deficiency anemia during early development. J. Nutr. 126, 693-701 (1996).

6. Georgieff, M. K. Long-term brain and behavioral consequences of early iron deficiency. Nutr. Rev. 69(Suppl 1), S43-S48 (2011)

7. Petry, N. et al. The proportion of anemia associated with iron deficiency in low, medium, and high human development index countries: a systematic analysis of national surveys. Nutrients 8, E693 (2016).

8. Park, S. et al. Mechanistic pathways from early gestation through infancy and neurodevelopment. Pediatrics 138, e20161843 (2016).

9. El-Farrash, R. A., Ismail, E. A. \& Nada, A. S. Cord blood iron profile and breast milk micronutrients in maternal iron deficiency anemia. Pediatr. Blood Cancer 58, 233-238 (2012).

10. Kumar, A., Rai, A. K., Basu, S., Dash, D. \& Singh, J. S. Cord blood and breast milk iron status in maternal anemia. Pediatrics 121, e673-e677 (2008).

11. Hay, G. et al. Predictors of serum ferritin and serum soluble transferrin receptor in newborns and their associations with iron status during the first $2 \mathrm{y}$ of life. Am. J. Clin. Nutr. 86, 64-73 (2007).

12. Abioye, A. I. et al. Anemia of inflammation during human pregnancy does not affect newborn iron endowment. J. Nutr. 148, 427-436 (2018).

13. Ziegler, E. E., Nelson, S. E. \& Jeter, J. M. Iron stores of breastfed infants during the first year of life. Nutrients 6, 2023-2034 (2014).

14. Wang, C. Y. \& Babitt, J. L. Hepcidin regulation in the anemia of inflammation. Curr. Opin. Hematol. 23, 189-197 (2016).

15. Nicolas, G. et al. Severe iron deficiency anemia in transgenic mice expressing liver hepcidin. Proc. Natl Acad. Sci. USA 99, 4596-4601 (2002).

16. Ganz, T. Hepcidin, a key regulator of iron metabolism and mediator of anemia of inflammation. Blood 102, 783-788 (2003).

17. Koenig, M. D., Tussing-Humphreys, L., Day, J., Cadwell, B. \& Nemeth, E. Hepcidin and iron homeostasis during pregnancy. Nutrients 6, 3062-3083 (2014).
18. Leenstra, T. et al. Schistosomiasis japonica, anemia, and iron status in children, adolescents, and young adults in Leyte, Philippines 1. Am. J. Clin. Nutr. 83, 371-379 (2006)

19. Leenstra, T. et al. Schistosoma japonicum reinfection after praziquantel treatment causes anemia associated with inflammation. Infect. Immun. 74, 6398-6407 (2006).

20. Friedman, J. F., Kanzaria, H. \& McGarvey, S. T. Human schistosomiasis and anemia: the relationship and potential mechanisms. Trends Parasitol. 21, 386-392 (2005).

21. Onguru, D. et al. Human schistosomiasis is associated with endotoxemia and Tolllike receptor 2- and 4-bearing B cells. Am. J. Trop. Med. Hyg. 84, 321-324 (2011).

22. Olveda, R. et al. Efficacy and safety of praziquantel for the treatment of human schistosomiasis during pregnancy: a phase 2 , randomised, double-blind, placebocontrolled trial. Lancet Infect. Dis. 16, 199-208 (2016).

23. WHO. Prevention and control of intestinal parasitic infections. World Health Organ Tech. Rep. Ser. 749, 1-86 (1987).

24. Coutinho, H. et al. Nutritional status and serum cytokine profiles in children, adolescents, and young adults with Schistosoma japonicum-associated hepatic fibrosis, in Leyte, Philippines. J. Infect. Dis. 192, 528-536 (2005).

25. FDA. Quantikine sTfR IVD Kit: 510(k) Substantial Equivalence Determination Decision Summary (US Food and Drug Administration, Silver Spring, MD, 2008).

26. WHO. Haemoglobin Concentrations for the Diagnosis of Anaemia and Assessment of Severity. Vitamin and Mineral Nutrition Information System (World Health Organization, Geneva, 2011).

27. WHO. Serum Ferritin Concentrations for the Assessment of Iron Status and Iron Deficiency in Populations. Vitamin and Mineral Nutrition Information System (World Health Organization, Geneva, 2011).

28. van den Broek, N., Letsky, E., White, S. \& Shenkin, A. Iron status in pregnant women: which measurements are valid? Br. J. Haematol. 103, 817-824 (1998).

29. Shin, D., Kim, H., Park, M., Suh, I. \& Shin, K. Utility of access soluble transferrin receptor (sTfR) and sTfR/log Ferritin Index in diagnosing iron deficiency anemia. Ann. Clin. Lab. Sci. 45, 396-402 (2015).

30. Phiri, K. S. et al. New cut-off values for ferritin and soluble transferrin receptor for the assessment of iron deficiency in children in a high infection pressure area. J. Clin. Pathol. 62, 1103-1106 (2009).

31. Coutinho, $\mathrm{H}$. et al. Pro-inflammatory cytokines and C-reactive protein are associated with undernutrition in the context of Schistosoma japonicum infection. Am. J. Trop. Med. Hyg. 75, 720-726 (2006).

32. Coutinho, H. M. et al. Nutritional status improves after treatment of schistosoma japonicum-infected children and adolescents. J. Nutr. 136, 183-188 (2006).

33. Raiten, D. et al. Executive summary--biomarkers of nutrition for development: building a consensus. Am. J. Clin. Nutr. 94, 633S-650SS (2011).

34. Scholl, T. O. Maternal iron status: relation to fetal growth, length of gestation, and iron endowment of the neonate. Nutr. Rev. 69, S23-S29 (2011).

35. Abioye, A. I. et al. Iron supplementation affects hematologic biomarker concentrations and pregnancy outcomes among iron-deficient Tanzanian women. J. Nutr. 146, 1162-1171 (2016).

36. Gambling, L. et al. Fetal iron status regulates maternal iron metabolism during pregnancy in the rat. Am. J. Physiol. Regul. Integr. Comp. Physiol. 296, R1063-R1070 (2009).

37. Lubach, G. \& Coe, C. Preconception maternal iron status is a risk factor for iron deficiency in infant rhesus monkeys (Macaca mulatta). J. Nutr. 136, 2345-2349 (2006).

38. Lozoff, B. et al. Low-dose iron supplementation in infancy modestly increases infant iron status at 9 mo without decreasing growth or increasing illness in a randomized clinical trial in rural China. J. Nutr. 146, 612-621 (2016).

39. Uyoga, M. et al. Duration of exclusive breastfeeding is a positive predictor of iron status in 6- to 10-month-old infants in rural Kenya. Matern. Child Nutr. 13, https:// doi.org/10.1111/mcn.12386 (2017). 